\section{Alteração do pH fecal de equinos pela administração de alcalinizantes orais}

\author{
Alteration of the fecal pH of the equines by oral administration \\ of alkalizing agents
}

\author{
Milena Alves Maia', Paulo de Tarso Landgraf Botteon²*, Bruno Ferreira Spindola ${ }^{3}$ \& \\ Rita de Cassia Campbell Machado Botteon ${ }^{2}$ \\ 'Medica veterinária. Clinica Veterinária Petlovers, Taubaté, SP, Brasil \\ 2Médicos veterinários, Drs. Departamento de Medicina e Cirurgia Veterinária - DMCV, Instituto de Veterinária - IV, Universidade \\ Federal Rural do Rio de Janeiro - UFRRJ, Seropédica, RJ, Brasil \\ ${ }_{3}^{3}$ Médico veterinário, Dr. Hospital Veterinário. Universidade Federal Rural do Rio de Janeiro - UFRRJ, Seropédica, RJ, Brasil
}

\section{Resumo}

Nos equinos, diversas doenças estão associadas ao consumo de dietas com níveis elevados de grãos ou carboidratos solúveis. O excesso destes alimentos, é comum a ocorrencia de fermentação lactica e acidose intestinal, provocando colicas e laminite. Este estudo teve como objetivo o uso de alcalinizantes diferentes e associações entre eles, em uma tentativa de reverter o processo e controle da acidose no intestino grosso de cavalos. 20 animais foram submetidos a quatro tratamentos com um controle, outro com Al2OH3, MgOH e, finalmente, a combinação destes. Os animais foram avaliados quanto a alterações no pH fecal e alterações clínicas decorrentes dos produtos administrados, como ocorencia de constipação ou diarréia, e desconforte abdominal. Conclui-se que a administração de hidroxido de magnésio $\left(\mathrm{Mg}(\mathrm{OH})_{2}\right.$ na dose de 20g/100 Kg de peso vivo e a associação de hidroxido de magnésio e hidróxido de aluminio $20 \mathrm{gl}(\mathrm{OH})_{3}+2 \mathrm{Og} \mathrm{Mg}(\mathrm{OH})_{2}, 100 \mathrm{Kg}$ de peso vivo, foram capazes de alterar significativamente $(\mathrm{P}<0,05) \mathrm{O}$ pH fecal, 24 horas após a administração. As doses empregadas nos três tratamentos com alcalinizantes, produziram um efeito catártico moderado, com diminuição da consistência fecal, fezes amolecidas, mas sem promover diarreia, ou desconforto.

Palavras-chave: acidose intestinal, alcalinizante, colica.

\begin{abstract}
In horses, many diseases are associated with the consumption of diets with high levels of grain or nonstructural carbohydrates. Excess of these foods, often con promotes lactic fermentation and intestinal acidosis, causing colic and laminitis. The addition of alkalizing in the diet does not have the same effect on horses as compared to ruminants. This study aimed to use different alkalizing and associations between them, in an attempt to reverse the process and control of acidosis in thelarge intestine of horses. 20 animals underwent four treatments with a control, one with $\mathrm{Al} 2(\mathrm{OH}) 3, \mathrm{Mg}(\mathrm{OH}) 2$, and finally a combination thereof. The animals were evaluated for changes in fecal $\mathrm{pH}$ and clinical changes resulting from the administered products such as the occurrence of constipation or diarrhea, and abdominal discomfort. It is concluded that administration of magnesium hydroxide $(\mathrm{Mg}(\mathrm{OH}) 2$ at a dose of $20 \mathrm{~g} / 100 \mathrm{~kg}$ body weight and the combination of magnesium hydroxide and aluminum hydroxide $20 \mathrm{gl}(\mathrm{OH}) 3+20 \mathrm{~g} \mathrm{Mg}(\mathrm{OH}) 2 / 100 \mathrm{~kg}$ live weight, were able to significantly $(\mathrm{P}<0,05)$ alter the fecal $\mathrm{pH}, 24$ hours after administration. The doses used in the three treatments with alkalinizing agents, produced a moderate cathartic effect, with decreased fecal consistency, faeces softened, but without promoting diarrhea, or discomfort.
\end{abstract}

Keywords: hindgut acidosis, alkalinizing agents, colic.

\section{Introdução}

Cavalos são herbívoros monogástricos, fisiologicamente adaptados para a digestão de grandes quantidades de forragens com alto teor de fibras. (Pagan et al., 2010). O intestino compreende a 20\% do peso vivo de equinos, devido principalmente aos compartimentos ceco e colo. Estes são seguimentos grandes onde ocorre a digestão fermentativa nos equídeos. Estudos a respeito dos padrões de comportamento dos equinos livres em pastagens demonstram que estes gastam de 10 a 16 horas por dia com o pastejo, com duração de 2 a 3 horas para cada refeição, separados por intervalos cursos para digestão, locomoção e outras atividades sociais (Tyler, 1972; Duncan, 1980;
Como citar: Maia, M. A., Botteon, P.T. L., Spindola, B. F., \& Botteon, R. C. C. M. (2017). Alteração do $\mathrm{pH}$ fecal de equinos pela administração de alcalinizantes orais. Brazilian Journal of Veterinary Medicine, 39(1), 1-6. doi: 10.29374/2527-2179. bjvm017

Fonte de financiamento: MAM é bolsista PIBIC/UFRRJ.

Conflito de interesses: Os autores declaram não haver conflito de interesses que precisam ser informados.

Recebido: Julho 03, 2016

Aceito: Novembro 12, 2017

O estudo foi realizado na Universidade Federal Rural do Rio de Janeiro - UFRRJ, Seropédica, RJ, Brasil.

\section{*Correspondência}

Paulo de Tarso Landgraf Botteon

Departamento de Medicina e Cirurgia Veterinária-DMCV, Instituto de Veterinária

- IV, Universidade Federal Rural do Rio de Janeiro - UFRRJ

$\mathrm{Km} 47, \mathrm{BR} 465$

CEP 23890-000 - Seropédica (SP), Brasil

E-mail: pbotteon@ufrrj.br 
Dittrich et al., 2007; Radünz, 2008; Santos et al., 2006; Zanine et al., 2006). O pastejo noturno é de 20 a 50\% do tempo de ingestão diária, influenciado pelas condições ambientais (Doreau et al., 1980; Dittrich et al., 2007). A limitação do tempo destinado ao pastejo pela manutenção de cavalos em cocheira, mesmo que somente no período noturno, diminui o tempo diário de alimentação, aumenta o tempo ocioso (Pond et al.,1993) e desencadeia aumento na frequência de estereotipias (Johnson et al., 1998), o que prejudica a saúde geral dos animais.

Estas observações representam um contraste marcante com o manejo alimentar de muitos cavalos domésticos, particularmente para os mantidos em confinamento. A ração ofertada contem quantidades substanciais de grãos ou produtos ricos em amido, com baixa proporção de matéria seca provenientes de carboidratos estruturais, quando comparados com cavalos mantidos em pastagens. Estas dietas, especialmente ricas em grãos e mudanças dietéticas recentes na alimentação, foram identificadas por estudos clínicos e epidemiológicos como um importante fator de risco para o desenvolvimento de cólica e laminite, as duas principais causas de mortalidade equina (Clarke et al., 1990; Hintz \& Cymbaluk, 1994; Hillyer \& Mair, 1997; Mair \& Hillyer, 1997; Tinker et al., 1997; Hudson et al., 2001). Propõe-se que quando os cavalos são introduzidos de repente a dietas contendo níveis elevados de carboidratos não estruturados (amido) (> 0,4\% do peso corporal), uma proporção substancial de amido atinge o intestino grosso (Potter et al., 1992; Lopes et al., 2004). Isto ocorre devido à capacidade limitada do cavalo em digerir completamente o amido no intestino delgado (Potter et al., 1992). Pesquisas recentes demonstram que dependendo da origem do amido, o escape para o intestino grosso é maior. Milho por exemplo não deve ser oferecido em uma única refeição em quantidades superiores a $2 \mathrm{~kg}$ para um cavalo de $500 \mathrm{~kg}$ já adaptado ao consumo de concentrados. Quando estes limites são ultrapassados há um aumento do risco de cólica, seja por uma mudança na quantidade, tipo de grão oferecido, adaptação lenta ou inadequada dos mecanismos para a digestão de amido no intestino delgado (Geor \& Haris, 2007).

Uma vez atingindo os compartimentos fermentativos do intestino, ceco e colón maior, alimentos ricos em carboidratos não estruturados sofrem rápida fermentação, o que resulta em elevação nos níveis de produção de ácidos graxos voláteis (AGV) e aumenta a produção deácido lático, provocando uma diminuição significativa do pH (Goodson et al., 1988; De Fombelle et al., 2001; Julliand et al., 2001). O ácido láctico é um ácido mais forte do que o AGV e podem causar irritação ou dano à mucosa intestinal. Em casos graves, ácido láctico pode contribuir entre 50\% e 90\% do total dos ácidos no intestino grosso (Van Soest, 1994). O acumulo destes produtos no intestino grosso pode desencadear efeitos secundários. A acidez provoca irritação e danos à mucosa intestinal. Além disso, promove a morte de bactérias que não resistem a este $\mathrm{pH}$, liberando lipopolissacarídeos bacterianos (LPS) ou endotoxinas, que podem ser absorvidas e promover danos teciduais decorrentes da endotoxemia. Esta condição de acidez intestinal irá também afetar o animal através de uma acidose sistêmica, provocando desconforto, cólicas e laminite (Hoffman, 2009). Outras alterações bioquímicas com a rápida fermentação do amido (ou fructanas) no intestino grosso podem incluir um aumento na produção de monoaminas vasoativas (por exemplo, tiramina, triptamina), endotoxinas e exotoxinas, todos os quais têm sido implicados na patogênese de laminite (Bailey et al., 2002; Botteon et al., 2008).

Estratégias para estabilizar o processo fermentativo, evitando-se variações importantes do $\mathrm{pH}$ intestinal podem evitar a ocorrência de complicações decorrentes de erros alimentares. Em bovinos, o principal método de controle da acidose ruminal emprega substancias alcalinizantes, visando ao tamponamento do conteúdo ruminal e impedindo o agravamento do quadro. Este trabalho busca avaliar a adição de alcalinizantes orais em equinos.

\section{Materiais e métodos}

Foram utilizados 20 equinos hígidos, mestiços de Mangalarga Marchador, com idade variando entre 18 e 24 meses, peso variando de 250 a $300 \mathrm{Kg}$, procedentes do rebanho da UFRRJ. Antes de iniciar o experimento, os animais foram avaliados, selecionados e, posteriormente, pesados e numerados de 01 a 20. Receberam água e feno de coast cross (cynodon dactylon) ad libitum e $15 \mathrm{~g}$ de sal mineral para equinos (Equifos 70 - Purina - Brasil). Passaram por controle de endo e ectoparasitos, administrando-se uma base conjugada de ivermectina e pomoatos de praziquantel e pirantel (Centurion-Valleé. Brasil) e deltametrina (Butox-Intervet AS. Brasil), respectivamente. 
O estudo foi conduzido em delineamento inteiramente casualizado com quatro tratamentos e cinco repetições $(n=5)$. O Grupo controle (GC) recebeu $1 \mathrm{~L}$ de água destilada, administrados por meio de sonda nasogástrica. O Grupo $(\mathrm{Mg})$ recebeu $20 \mathrm{~g} / 10 \mathrm{O} \mathrm{Kg}$ de peso de $\mathrm{Mg}(\mathrm{OH})_{2}$ diluído em $1 \mathrm{~L}$ de água destilada, administrados por meio de sonda nasogástrica. O grupo (Al) recebeu 20g/10O Kg de peso de $\mathrm{Al}(\mathrm{OH})_{3}$ diluído em $1 \mathrm{~L}$ de água destilada, administrados por meio de sonda nasogástrica. O grupo ( $\mathrm{MgAl}), 20 \mathrm{~g}$ de $\mathrm{Mg}(\mathrm{OH})_{2} / 100 \mathrm{Kg}$ + $20 \mathrm{~g}$ de $\mathrm{Al}(\mathrm{OH})_{3} / 100 \mathrm{Kg}$ diluídos em um litro de água destilada, administrados por meio de sonda nasogástrica.

As avaliações foram realizadas no momento imediatamente antes da intubação nasogástrica e administração da solução, com o animal em repouso, e nos momentos 6 h, 12 h, 24 h, 36 h, 48 h e 72 h após a administração do alcalinizante ou de água destilada, respectivamente nos grupos tratados e controle, totalizando sete amostras em cada repetição.

Amostras de fezes foram coletadas diretamente da ampola retal, nos intervalos já mencionados, utilizando saco plástico e uma pequena quantidade de óleo mineral como lubrificante.

$\mathrm{O}$ pH fecal foi determinado imediatamente após a coleta diretamente da ampola retal, utilizando-se uma proporção de $30 \mathrm{~g}$ de fezes, que foi diluída e homogeneizada em $30 \mathrm{ml}$ de água destilada. Depois de realizada a homogeneização a solução foi filtrado com gaze e funil de vidro coletando-se o volume de aproximadamente $20 \mathrm{ml}$ de amostra a qual foi medido o pH usando um phmetro digital, segundo a metodologia descrita por Silva (1998).

Os animais foram avaliados clinicamente, nos intervalos acima mencionados, avaliando-se comportamento, atitude por inspeção, apetite, ingestão por inspeção; coloração de mucosas, por inspeção; o tempo de preenchimento capilar, por compressão digital, seguida de observação do tempo em segundos de reestabelecimento da vascularização; o turgor cutâneo, determinado por palpação; a temperatura retal foi aferida por termômetro digital; frequência cardíaca, frequência respiratória, murmúrio vesicular e motilidade intestinal, por auscultação e avaliados quanto à presença e aspecto das fezes.

A consistência fecal foi avaliada e classificada em escores, em escala de 1 a 4, onde fezes ressecadas (R) corresponderam a escore (1); fezes normais (N) (cíbalas arredondadas de consistência firme) corresponderam ao escore (2); fezes amolecidas (M) (ou pastosas) corresponderam ao escore (3) e fezes diarreicas (D), corresponderam ao escore (4).

Os dados foram analisados quanto à distribuição normal pelo teste Shapiro-Wilk. Em seguida, foram submetidos ao teste Anova para medidas repetidas (RM-ANOVA). No pós-teste, para comparação múltipla de médias, foi aplicado o teste de Bonferroni, com 95\% de significância. Dados não paramétricos foram avaliados pelo teste de Friedman.

\section{Resultados e discussão}

Os valores de temperatura retal mantiveram-se dentro dos limites fisiológicos estabelecidos para a espécie, observamos média e desvio padrão de 38,11 $\pm 0,23$, sem diferenças entre os grupos. Os valores de frequência cardíaca observados também mantiveram-se dentro dos limites fisiológicos para a espécie e para a idade, não apresentando diferenças entre os grupos, foram verificados valores médios de 57,3 bpm com desvio padrão de 3.6 bpm. A frequência cardíaca, é um parâmetro importante para avaliação de dor ou desconforto abdominal. A manutenção da condição clinica durante toda a avaliação é um indicativo de que os produtos administrados não causaram desconforto aos animais, contudo, este parâmetro não deve ser considerado isoladamente, alterações comportamentais e interação com o ambiente, são parâmetros igualmente importantes nesta avaliação (Taffarel, 2013). Neste estudo estas alterações não foram observadas durante o exame clínico.

Quanto às alterações do pH fecal, conforme pode ser observado na Tabela 1, houve diferença significativa entre os valores de $\mathrm{pH}$ fecal para os tratamentos e os tempos avaliados, com aumento do $\mathrm{pH}$ fecal decorrente da administração de $\mathrm{MgOH}_{2}$ e $\mathrm{AlOH}_{3}+\mathrm{MgOH}_{2}$, este efeito foi observado 24 horas após a administração do alcalinizantes. Willard et al. 1997 demonstraram a eficácia da administração de bicarbonato através de fístula cecal, Souza (2007) trabalhando com animais submetidos a indução experimental de laminite por sobrecarga de carboidratos, observou uma queda menos acentuada do pH cecal. Porém Souza (2007) trabalhou com animais fistulados e aplicou alcalinizantes $\left(\mathrm{AlOH}_{3 ;} ; 3,5 \mathrm{~g}+\mathrm{MgOH}_{2} ; 65,6 \mathrm{~g}+\right.$ dimeticona; $\left.1,2 \mathrm{~g}\right)$ e avaliou o $\mathrm{pH}$ diretamente no ceco dos eqüinos. Os resultados obtidos neste trabalho divergem de Rowe et al. (1995), que 
afirma que a adição pura e simples de um alcalinizante à dieta dos equinos não surte efeito, pois o agente é absorvido no intestino delgado não sendo disponibilizado para a ação no intestino grosso. Recentemente, Taylor et al. (2014) administraram bicarbonato oralmente, $1 \mathrm{~g} / \mathrm{kg}$ de peso corporal, e observaram elevação do pH cecal entre 6 e 12 horas após a administração, concluindo que a administração de bicarbonato oral pode ser benéfica para o tamponamento de acidose cecal, o que corrobora os resultados obtidos neste experimento, porém com alcalinizantes diferentes.

A avaliação do pH fecal éuma medida indireta das alterações ocorridas no trato digestivo, contudo não é invasiva e permite sua utilização rotineira na prática clínica. Em bovinos, o principal método de controle da acidose ruminal emprega substancias alcalinizantes, visando ao tamponamento do conteúdo ruminal e impedindo o agravamento do quadro (Radostits et al., 2002).

A consistência das fezes também foi avaliada após a administração dos sais de Mg e Al (Tabela 2). Um dos inconvenientes do uso de sais de Mg está na possibilidade de provocarmos uma diarreia em função do efeito catártico deste sal (Hintz \& Schryver, 1972). Sais de Mg causam diarreia e sais de Al causam constipação razão pela qual podem ser usados em associação para preservação da função intestinal normal (Colin-Jones, 1990). Sais de magnésio são frequentemente usados como purgantes salinos. Normalmente, apenas 20\% do magnésio é sistemicamente absorvido e eliminado pelos rins. Se a absorção é excessiva ou renal eliminação é prejudicada, então grave hipermagnesemia e alcalose metabólica pode se desenvolver (Schryver et al., 1987; Hintz \& Cymbaluk, 1994). Neste estudo, observamos diminuição da consistência fecal em nos grupos $\mathrm{Mg}(\mathrm{OH})_{2^{\prime}} \mathrm{Al}(\mathrm{OH})_{3}$ e $\mathrm{Al}(\mathrm{OH})_{3}+\mathrm{Mg}(\mathrm{OH})_{2^{\prime}}$ não havendo diferença significativa entre estes grupos, mas todos diferiram em relação ao grupo Controle (Valor $\mathrm{P}=0,0146$ ). A diminuição da consistência

Tabela 1. Valores médios de $\mathrm{pH}$ fecal, nos diferentes tratamentos: Grupo controle, $\mathrm{Mg}(\mathrm{OH})_{2} ; \mathrm{Al}(\mathrm{OH})_{3}$ e $\mathrm{Mg}(\mathrm{OH})_{2}+\mathrm{Al}(\mathrm{OH})_{3}$.

\begin{tabular}{ccccc}
\hline Tempo & Controle & $\mathbf{M g}(\mathbf{O H})_{2}$ & $\mathbf{A l}(\mathbf{O H})_{3}$ & $\mathbf{A l}(\mathbf{O H})_{3}+\mathbf{M g}(\mathbf{O H})_{2}$ \\
\hline 0 & $6,87 \mathrm{Aa}$ & $6,87 \mathrm{Aa}$ & $6,79 \mathrm{Aa}$ & $6,91 \mathrm{Aa}$ \\
6 & $6,87 \mathrm{Aa}$ & $6,67 \mathrm{Aa}$ & $6,47 \mathrm{Aa}$ & $6,80 \mathrm{Aa}$ \\
12 & $6,68 \mathrm{Aa}$ & $6,71 \mathrm{Aa}$ & $6,73 \mathrm{Aa}$ & $6,78 \mathrm{Aa}$ \\
24 & $6,82 \mathrm{Aa}$ & $7,39 \mathrm{Bb}$ & $6,68 \mathrm{Aa}$ & $7,52 \mathrm{Bb}$ \\
36 & $6,95 \mathrm{Aa}$ & $7,06 \mathrm{Aa}$ & $6,80 \mathrm{Aa}$ & $7,11 \mathrm{Aa}$ \\
48 & $6,91 \mathrm{Aa}$ & $6,86 \mathrm{Aa}$ & $6,70 \mathrm{Aa}$ & $6,69 \mathrm{Aa}$ \\
60 & $6,78 \mathrm{Aa}$ & $6,85 \mathrm{Aa}$ & $6,63 \mathrm{Aa}$ & $6,97 \mathrm{Aa}$ \\
\hline
\end{tabular}

Médias seguidas pelas mesmas letras minúsculas nas linhas e maiúsculas nas colunas, não diferem significativamente entre si pelo teste de Tukey a $5 \%$

Tabela 2. Frequência observada da consistência fecal, Ressecada (R), Normal (N), Amolecida (M), Diarreica (D) em cada um dos tratamentos administrados por sonda nasogástrica diluídos em 11 de água.

\begin{tabular}{|c|c|c|c|c|c|c|c|c|c|c|c|c|c|c|c|c|}
\hline \multirow{2}{*}{ Tempo } & \multicolumn{4}{|c|}{ Controle } & \multicolumn{4}{|c|}{$\mathrm{Mg}(\mathrm{OH})_{2}$} & \multicolumn{4}{|c|}{$\mathrm{Al}(\mathrm{OH})_{3}$} & \multicolumn{4}{|c|}{$\mathrm{Mg}(\mathrm{OH})_{2}+\mathrm{AL}(\mathrm{OH})_{3}$} \\
\hline & $\mathbf{R}$ & $\mathbf{N}$ & $\mathbf{M}$ & D & $\mathbf{R}$ & $\mathbf{N}$ & $\mathbf{M}$ & D & $\mathbf{R}$ & $\mathbf{N}$ & M & D & $\mathbf{R}$ & $\mathbf{N}$ & $\mathbf{M}$ & D \\
\hline 0 & 0 & 5 & 0 & 0 & 0 & 5 & 0 & 0 & 0 & 5 & 0 & 0 & 0 & 5 & 0 & 0 \\
\hline $6 \mathrm{~h}$ & 0 & 5 & 0 & 0 & 0 & 5 & 0 & 0 & 0 & 3 & 3 & 0 & 0 & 4 & 1 & 0 \\
\hline $12 \mathrm{~h}$ & 0 & 4 & 1 & 0 & 0 & 5 & 0 & 0 & 0 & 5 & 0 & 0 & 0 & 4 & 1 & 0 \\
\hline $24 \mathrm{~h}$ & 0 & 4 & 1 & 0 & 0 & 1 & 4 & 0 & 0 & 3 & 2 & 0 & 0 & 1 & 4 & 0 \\
\hline $36 \mathrm{~h}$ & 0 & 5 & 0 & 0 & 0 & 4 & 1 & 0 & 1 & 3 & 1 & 0 & 0 & 2 & 3 & 0 \\
\hline $48 \mathrm{~h}$ & 0 & 5 & 0 & 0 & 0 & 3 & 2 & 0 & 0 & 1 & 4 & 0 & 0 & 2 & 3 & 0 \\
\hline \multirow[t]{2}{*}{$72 \mathrm{~h}$} & 0 & 5 & 0 & 0 & 0 & 4 & 1 & 0 & 0 & 3 & 2 & 0 & 0 & 3 & 2 & 0 \\
\hline & \multicolumn{4}{|c|}{$\mathrm{a}$} & \multicolumn{4}{|c|}{ b } & \multicolumn{4}{|c|}{$b$} & \multicolumn{4}{|c|}{$b$} \\
\hline
\end{tabular}

Parâmetro avaliado pelo teste de Friedman. Entre os tratamentos, valores seguidos pelas mesmas letras, ou sem elas não diferem estatisticamente, Valor $\mathrm{P}=0.0146$. 
fecal foi observada a parir de 24 h após a administração dos alcalinizantes, com diferença significativa em relação ao momento zero (Valor $\mathrm{P}=0,040$ ) e o momento 48h (Valor $\mathrm{P}=0,047$ ). Contudo, observou-se apenas amolecimento das fezes. Diarreia não foi observada em nenhum animal. O efeito de constipação relatado para $\mathrm{Al}(\mathrm{OH})_{3^{\prime}}$ também não foi verificado nos animais deste estudo, na dose empregada.

\section{Conclusão}

Dentre os produtos testados, o $\mathrm{Mg}(\mathrm{OH})_{2}$ isoladamente ou em associação $\mathrm{Al}(\mathrm{OH})_{3}+\mathrm{Mg}(\mathrm{OH})_{2}$, foram eficientes em promover a alcalinização do bolo fecal. As doses empregadas nos três tratamentos com alcalinizantes, produziram um efeito catártico moderado, com diminuição da consistência fecal, fezes amolecidas, mas sem promover diarreia, ou desconforto.

\section{Agradecimentos}

À Faperj pelo apoio financeiro a este trabalho. Ao CNPq, pelo apoio através da bolsa de iniciação cientifica.

\section{Referências}

Bailey, S. R., Rycroft, A., \& Elliott, J. (2002). Production of amines in equine cecal contents in an in vitro model of carbohydrate overload1. Journal of Animal Science, 80(10), 2656-2662. PMid:12413088.

Botteon, P. T. L., Ferraz, V. P., Glória, M. B. A., Melo, M. M., Paes Leme, F. O., Faleiros, R. R., \& Alves, G. E. S. (2008). Concentrações plasmáticas de triptamina, tiramina e feniletilamina em eqüinos sob efeitos de sobrecarga de carboidratos e antiinflamatórios não esteroidais. Pesquisa Veterinária Brasileira, 28, 299-302.

Clarke, L. L., Roberts, M. C., \& Argenzio, R. A. (1990). Feeding and digestive problems in horses. Physiologic responses to a concentrated meal. The Veterinary Clinics of North America. Equine Practice, 6(2), 433-450. http://dx.doi.org/10.1016/S0749-0739(17)30550-3. PMid:2202501.

Colin-Jones, D. G. (1990). Acid suppression - how much is needed. British Medical Journal, 301(6752), 564-565. http://dx.doi.org/10.1136/bmj.301.6752.564. PMid:1978697.

De Fombelle, A., Julliand, V., Drogoul, C., \& Jacotot, E. (2001). Feeding and microbial disorders in horses: 1. Effects of an abrupt incorporation of two levels of barley in a hay diet on microbial profile and activities. Journal of Equine Veterinary Science, 21(9), 439-445. http://dx.doi.org/10.1016/50737-0806(01)70018-4.

Dittrich, J. R., Faccio Carvalho, P. C., Moraes, A., Oliveira, E. B., Dittrich, R. L., Oikawa, M., Souza, F. T. V, \& Santos, F. (2007). Comportamento ingestivo de equinos em pastejo sobre diferentes dosséis. Ciência Animal Brasileira, 8, 87-94.

Doreau, M., Martin-Rosset, W., \& Petit, D. (1980). Nocturnal feeding activities of horses at pasture. Annales de Zootechnie, 29, 299-304. http://dx.doi.org/10.1051/animres:19800305.

Duncan, P. (1980). Time-budgets of Camargue horses. II. Time-budgets of adult horses and weaned sub-adults. Behaviour, 72(1), 26-49. http://dx.doi.org/10.1163/156853980x00023.

Geor, R. J., \& Harris, P. A. (2007). How to minimize gastrointestinal disease associated with carbohydrate nutrition in horses. AAEP Proceedings, 53, 178-185.

Goodson, J., Tyznik, W. J., Cline, J. H., \& Dehority, B. A. (1988). Effects of an abrupt diet change from hay to concentrate on microbial numbers and physical environment in the caecum of the pony. Applied and Environmental Microbiology, 54(8), 1946-1950. PMid:3178206.

Hillyer, M. H., \& Mair, T. S. (1997). Recurrent colic in the mature horse: a retrospective review of 58 cases. Equine Veterinary Journal, 29(6), 421-424. http://dx.doi.org/10.1111/j.2042-3306.1997.tb03152.x. PMid:9413712.

Hintz, H. F., \& Cymbaluk, N. F. (1994). Nutrition of the horse. Annual Review of Nutrition, 14(1), 243-267. http:/l dx.doi.org/10.1146/annurev.nu.14.070194.001331. PMid:7946520.

Hintz, H. F., \& Schryver, H. F. (1972). Magnesium metabolism in the horse. Journal of Animal Science, 35(4), 755759. http://dx.doi.org/10.2527/jas1972.354755x. PMid:5075819.

Hoffman, R. M. (2009). Carbohydrate metabolism and metabolic disorders in horses. Revista Brasileira de Zootecnia, 38 (Suppl.), 270-276. http://dx.doi.org/10.1590/S1516-35982009001300027.

Hudson, J. M., Cohen, N. D., Gibbs, P. G., \& Thompson, J. A. (2001). Feeding practices associated with colic in horses. Journal of the American Veterinary Medical Association, 219(10), 1419-1425. http://dx.doi.org/10.2460/ javma.2001.219.1419. PMid:11724182.

Johnson, K. G., Tyrrell, J., Rowe, J. B., \& Pethick, D. W. (1998). Behavioural changes in stabled horses given nontherapeutic levels of virginiamycin. Equine Veterinary Journal, 30(2), 139-143. http://dx.doi.org/10.1111/j.2042-3306.1998. tb04473.x. PMid:9535070. 
Julliand, V., De Fombelle, A., Drogoul, C., \& Jacotot, E. (2001). Feeding and microbial disorders in horses: Part 3-Effects of three hay: grain ratios on microbial profile and activities. Journal of Equine Veterinary Science, 21(11), 543-546. http://dx.doi.org/10.1016/S0737-0806(01)70159-1.

Lopes, M. A. F., White, N. A., Donaldson, L., Crisman, M. V., \& Ward, D. L. (2004). Effects of enteral and intravenous fluid therapy, magnesium sulfate, and sodium sulfate on colonic contents and feces in horses. American Journal of Veterinary Research, 65(5), 695-704. https://doi.org/10.2460/ajvr.2004.65.695

Mair, T. S., \& Hillyer, M. H. (1997). Chronic colic in the mature horse: Aretrospective review of 106 cases. Equine Veterinary Journal, 29(6), 415-420. http://dx.doi.org/10.1111/j.2042-3306.1997.tb03151.x. PMid:9413711.

Pagan, J. D., Lawrence, T. J., \& Lawrence, L. A. (2010, September). Feeding protected sodium bicarbonate attenuates hindgut acidosis in horses fed a high-grain ration. In The Impact of Nutrition on the Health and Welfare of Horses: No. 128. 5th European Workshop Equine Nutrition (pp.19-22). Cirencester, United Kingdom: Wageningen Academic Pub.

Pond, K. R., Dugan, D. K., Barnett, D. T., Burns, J. C., \& Fisher, D. S. (1993, January 21-23). Grazing behavior of mares and steers on orchardgrass and mares on common bermuda grass. In Proceedings of the 13th Equine Nutrition and Physiology Symposium (pp. 263). Gainesville, Florida: Institute of Food and Agricultural Sciences, University of Florida.

Potter, G. D., Arnold, F. F., Householder, D. D., Hansen, D. H., \& Brown, K. M. (1992). Digestion of starch in the small or large intestine of the equine. Pferdeheilkunde, 1(4), 107-111.

Radostits, O. M., Gay, C. C., Blood, D. C., Hinchcliff, K. W., \& McKenzie, R. A. (2002). Clínica Veterinária: um tratado de doenças dos bovinos, ovinos, suínos, caprinos e eqüinos (1737 p.). Rio de Janeiro: Guanabara Koogan.

Radünz, E. (2008). A estrutura de gramíneas do gênero Cynodon eo comportamento ingestivo de eqüinos. (Dissertação de mestrado). Universidade Federal do Paraná, Curitiba.

Rowe, J. B., Pethick, D. W., \& Johnson, K. G. (1995). Controlling acidosis in the equine hindgut. Recent Advances in Animal Nutrition in Australia, 136-142.

Santos, E. M., Zanine, A. M., Parente, H. N., Ferreira, D. J., Almeida, F. Q., \& Cecon, P. R. (2006). Comportamento ingestivo de eqüinos em pastagens de grama batatais (Paspalum notatum) e braquiarinha (Brachiaria decumbens) na região centro-oeste do Brasil. Ciência Rural, 36(5), 1565-1569. http://dx.doi.org/10.1590/ S0103-84782006000500035.

Schryver, H. F., Parker, M. T., Daniluk, P. D., Pagan, K. I., Williams, J., Soderholm, L. V., \& Hintz, H. F. (1987). Salt consumption and the effect of salt on mineral metabolism in horses. The Cornell Veterinarian, 77(2), 122-131. PMid:3568683.

Silva, D. J. (1998). Análise de alimentos - métodos químicos e biológicos (2. ed., 165p.). Viçosa: UFV-Imprensa Universitária.

Souza, A. H. (2007). Tamponamento cecal: aspectos clínico, fisiopatológico e terapêutico na laminite experimental, em eqüinos (Tese de doutorado). Faculdade de Ciências Agrárias e Veterinárias, Universidade Estadural Paulista, Jaboticabal.

Taffarel, M. O. (2013). Proposição de escala clínica para avaliação da dor em equinos (Tese de doutorado). Faculdade de Medicina Veterinária e Zootecnia de Botucatu, Universidade Estadual Paulista Júlio de Mesquita Filho, Botucatu.

Taylor, E. A., Beard, W. L., Douthit, T., \& Pohlman, L. (2014). Effect of orally administered sodium bicarbonate on caecal pH. Equine Veterinary Journal, 46(2), 223-226. http://dx.doi.org/10.1111/evj.12111. PMid:23738517.

Tinker, M. K., White, N. A., Lessard, P., Thatcher, C. D., Pelzer, K. D., Davis, B., \& Carmel, D. K. (1997). Prospective study of equine colic risk factors. Equine Veterinary Journal, 29(6), 454-458. http://dx.doi.org/10.1111/j.2042-3306.1997. tb03158.x. PMid:9413718.

Tyler, S. J. (1972). The behaviour and social organization of the New Forest ponies. Animal Behaviour Monogastric, 5, 85-96.

Van Soest, P. J. (1994). Nutritional ecology of the ruminant. London: Cornell University Press.

Zanine, A. M., Santos, E. M., Parente, H. N., Ferreira, D. F., \& Almeida, F. Q. (2006). Diferenças entre sexos para as atividades de pastejo de equinos no nordeste do Brasil. Archivos de Zootecnia, 55(210), 139-147. 